\title{
A New Number Theory: Considerations about the (3-n)d Algebra
}

\section{Sonaglioni $L^{*}$}

Free Professionist, Italy

\section{Abstract \\ General considerations about this (3-n)d algebra.}

\section{Introduction}

About 3 years ago, we have seen that we can build a commutative (3-n)d algebra [3] quite similar to the standard $2 \mathrm{~d}$ complex algebra; open arguments are lacks about derivatives and integrals, because as we have seen in paper [2] this algebra is not distributive. What can we do with this algebra?

The non distributive property does not permit and easy analytical exam of a problem (in general), but this is not a limitation if the approach is an applied computational study [2].

For the (3-n)d algebra, in general $\frac{d f(s)}{d s}$ does not exist, also a (3-n) $\mathrm{d}$ integral $\int f(s) . d s$ depends on the path, that means it couldn't calculate a primitive of $f(s)$ (note that $f(s) . d s$ is a (3-n)d product. As we have seen in paper [3].

A point in the $3 \mathrm{~d}$ space [1] can always be written as

$$
\begin{aligned}
& P(r, \alpha, \beta)=r(x, y, x) \cdot e^{\vec{j} \cdot \alpha+\vec{k} \cdot \beta} \\
& r(x, y, z)=\sqrt{x^{2}+y^{2}+z^{2}}
\end{aligned}
$$

The operator

$$
\psi(\alpha, \beta)=e^{j \cdot \alpha+\vec{k} \cdot \beta}=\cos (\alpha) \cdot \cos (\beta)+\vec{j} \cdot \sin (\alpha) \cdot \cos (\beta)+\vec{k} \cdot \sin (\beta)
$$

can be seen as a $3 \mathrm{~d}$ number function that plots a surface sphere of radius 1 around the origin, while $r(x, y, z) \in \Re$ is a standard real function and represent the modulus of the vector identified by the tern $(x, y, z)$.

It's quite simple to demonstrate that $\frac{\partial^{2} \psi}{\partial \alpha \cdot \partial \beta}=\frac{\partial^{2} \psi}{\partial \beta \cdot \partial \alpha}$

So the $\psi(\alpha, \beta)$ operator is differentiable.

In an applied computational approach, given an algebraic definition of a $f(s)$ (as for example a polynomial function), we can always study the function in polar notation or in algebraic notation:

$$
f(s)=f_{x}+\vec{j} \cdot f_{y}+\vec{k} \cdot f_{z} \Leftrightarrow f(r, \alpha, \beta)=r\left(f_{x}, f_{y}, f_{z}\right) \cdot e^{\vec{j} \cdot \alpha+\vec{k} \cdot \beta}
$$

and, because the $\psi(\alpha, \beta)$ operator is differentiable, also $f(s)$ is always differentiable (if $r\left(f_{x} f_{y} f_{z}\right)$ is differentiable).

This mean we can always calculate the gradient of a (3-n)d function, the divergence of a (3-n)d function, the rotor of a $3 \mathrm{~d}$ function, etc. and in general we can calculate partial derivatives of a generic multi dimensions $f(s)$ along any direction.

About the integrals, the main question is: what is the goal?

If the goal is to calculate a volume or a surface, in paper [1] we have seen there are no problems.

We can always calculate the elementary volume or elementary

surface along any directions of the operator $\psi(\alpha, \beta)=e^{\vec{j} \cdot \alpha+\vec{k} \cdot \beta}$, because $f(s) \Leftrightarrow f(r, \alpha, \beta)=r\left(f_{x}, f_{y}, f_{z}\right) \cdot e^{j \cdot \alpha+\vec{k} \cdot \beta}$ we can always estimate $d r$ by starting from any point in the $3 \mathrm{~d}$ space along any direction. As we have seen in paper [1] we can always estimate the elementary cube by changing the coordinates (see the function Project_A_on_B(..) in the 3d-4d appendix code papers [2], [3] or the elementary surface.

As for example, let us consider a generic surface, a $R^{2} \rightarrow R^{3}$ transformation:

$$
S(u, v)=f_{x}(u, v)+\vec{j} \cdot f_{y}(u, v)+\vec{k} \cdot f_{z}(u, v)
$$

The area of the surface is:

$$
A_{S}=\int_{D}\left|\frac{\partial S}{\partial u} \times \frac{\partial S}{\partial v}\right| \cdot d u \cdot d v
$$

Where $\mathrm{D}$ is the domain of $(u, v)$

The integral (3) can be easily computed in the same way as we do with the standard $3 \mathrm{~d}$ algebra in a convenient symbolic form.

For more dimension (4 and more), $\psi(\alpha, \beta)$ is still differentiable, and the consideration of above are the same; of course, in this case, the elementary volume is a hyper-elementary volume, the elementary surface is a hyper-elementary surface, and so on.

The operator $\psi(\alpha, \beta, \ldots)$ can be seen as a $4 \mathrm{~d}$ (or more dimensions) operator that plots a hyper surface sphere of radius 1 around the $4 \mathrm{~d}$ (or more dimensions) origin space.

A last consideration: we are talking about a (3-n) d commutative algebra, where the (3-n) d sum and product are well defined (it is well defined the algebraic product, see paper [3]), and where we have seen that the (3-n) d functions are always differentiable and the computational integrals are always possible.

For these reasons I suppose it is possible to define the discrete and the standard Fourier and Laplace transformations for a (3-n) d time function, but I really can't it imagine if this can be, in concrete, useful or not. *Corresponding author: Sonaglioni L, Free Professionist, Italy, Tel: 388-0579470;
E-mail: luca.sonaglioni@hotmail.com

Received November 19, 2018; Accepted November 24, 2018; Published November 30, 2018

Citation: Sonaglioni L (2018) A New Number Theory: Considerations about the (3-n)d Algebra. J Appl Computat Math 7: 427. doi: 10.4172/2168-9679.1000427

Copyright: (c) 2018 Sonaglioni L. This is an open-access article distributed under the terms of the Creative Commons Attribution License, which permits unrestricted use, distribution, and reproduction in any medium, provided the original author and source are credited. 
Citation: Sonaglioni L (2018) A New Number Theory: Considerations about the (3-n)d Algebra. J Appl Computat Math 7: 427. doi: 10.4172/21689679.1000427

Page 2 of 2

\section{Conclusions}

Paper [1] has introduced the basis of a new multi dimension commutative algebra that may be a good extension of the standard $2 \mathrm{~d}$ complex algebra.

This algebra permit to treat multi dimensions problems exactly in the same way we use to do actually with a simplified symbolic form, and with the extension of the definition of the product as number product; also the extensions of the multi dimensions external and internal product, comply with the standard operators used in the standard $3 \mathrm{~d}$ problems.
For multi dimensions numeric problems, they are also well defined the extended concepts of the sum and product, the exponential function (the $\psi(\alpha, \beta, .$.$) operator) and the log function. (See paper [2]).$

\section{References}

1. Sonaglioni L (2015) A New Number Theory. J Appl Computat Math.

2. Sonaglioni L (2015) A New Number Theory-Algebra Analysis II. J Appl Computat Math.

3. Sonaglioni L (2016) A New Number Theory-Algebra Analysis II. J Appl Computat Math.

4. Walker MJ (1956) Quaternions as 4-Vectors. Am J Phys 24: 515. 